\section{¿Existen relaciones entre los factores ambientales rurales y la salud reproductiva en la Pampa Húmeda Argentina?}

\author{
Is there any relationship between rural \\ environmental factors and reproductive health in \\ the Pampa Humeda in Argentina?
}

\author{
Alejandro Oliva ${ }^{1,2}$ \\ Ricardo Biasatti 1,3 \\ Silvia Cloquell 3 \\ Cristina González 4 \\ Susana Olego 5 \\ Alberto Gelin †
}

${ }_{1}$ Centro de Investigaciones en Biodiversidad y Ambiente, Rosario, Argentina. 2 Hospital Italiano de Rosario, Rosario, Argentina. 3 Universidad Nacional de Rosario, Rosario, Argentina. 4 Instituto Nacional de Tecnología Agropecuaria Rosario, Argentina. ${ }^{5}$ Federación Agraria Argentina, Rosario, Argentina. + Fallecido.

Correspondencia A. Oliva

Hospital Italiano de Rosario. Virasoro 1249, Rosario, Argentina.

andrologia@hig.org.ar

\section{Abstract}

The relationship between environmental factors and health is well known. Rural environmental influences on reproductive health have been properly proved, both in animals and humans. In Latin America, few studies have been conducted in this area. The current project is based on the description of relationships between reproductive health and environmental factors in rural populations, characterized by specific environmental characteristics. Three variables were evaluated: male-to-female birth ratio, male urogenital malformations (cryptorchidism and hypospadias), and endocrine-related cancer incidence. Five rural communities in the Pampa Humeda in Argentina were selected, and the data were compared to the national mean. Biomedical data and environmental risk factors were correlated through a geographic information system. The ratio of male to female births did not show any differences. Malformations showed very significant differences. Endocrinerelated cancers showed higher incidence rates compared to the national mean, particularly in some communities. In conclusion, there is a relationship between environmental factors and reproductive health conditions in this region.

Reproductive Medicine; Environment and Public Health; Rural Population

\section{Introducción}

En los últimos años diferentes observaciones, poblacionales y profesionales, han llamado la atención sobre la percepción de una mayor incidencia de malformaciones y cánceres en diferentes comunidades rurales situadas en la región de la Pampa Húmeda, perteneciente a la de la Provincia de Santa Fe, Argentina, generando una preocupación a nivel de salud pública.

En las últimas décadas ha quedado demostrado que las actividades humanas, como las industriales y agro-productivas, influencian las condiciones de salud-enfermedad. En particular, es sabido que una de las áreas de impacto es el de la salud reproductiva, particularmente malformaciones y cánceres hormono-dependientes, que ha sido estudiada ampliamente en los países desarrollados 1 .

En la década pasada un grupo de ecologistas, epidemiólogos, endocrinólogos y toxicólogos han llamado la atención sobre el potencial peligro de los efectos que los disruptores endocrinos - sustancias capaces de interferir con la fisiología hormonal - podrían tener sobre los humanos y otras especies animales. La hipótesis que fue lanzada es sobre ciertos químicos que podrían perturbar los sistemas endocrinos animales en distintos niveles 2 .

Las consecuencias de la exposición a diferentes agro-químicos continúa siendo uno de los mayores problemas de salud en comunida- 
des rurales, es por ello que las investigaciones con participación comunitaria son la mejor forma de poder llevar adelante acciones de identificación, diagnóstico y gestión de este mencionado riesgo ${ }^{3}$. Recientemente, ha sido publicado un extenso documento de la Royal Commission on Environmental Pollution del Reino Unido, en el que se ha establecido la prioridad para llevar adelante investigaciones relacionadas con pesticidas y salud rural, mostrando los diferentes aspectos que pudieran estar involucrados en el impacto de factores ambientales rurales sobre la salud humana 4 .

Las funciones reproductivas, tanto femeninas como masculinas, son altamente sensitivas a diferentes agentes químicos y/o físicos, generados en la actividad agrícola 5 .

El impacto sobre el sistema reproductivo masculino es actualmente motivo de un fuerte debate, debido a reportes que sugieren evidencias de un aumento en alteraciones a nivel del sistema genital masculino. Esto incluye un aumento de la incidencia de: cáncer de testículo (en hombres menores de 40 años); de algunas malformaciones del mencionado tracto (testículosno-descendidos e hipospadias) 6; disminución en la calidad seminal 7; así como mayor riesgo de disfunción eréctil 8 .

La salud reproductiva femenina también ha sido relacionada con el impacto de factores ambientales. Inicialmente, fue el cáncer de mama en mujeres pre-menopáusicas 9; la relación del cuadro de endometriosis con la presencia de dioxinas en el ambiente 10; y, finalmente, en los últimos años algunas publicaciones han resaltado la relación entre ambiente rural y fenómenos de precocidad sexual femenina 11 .

Diferentes estudios realizados sobre poblaciones rurales han demostrado que la incidencia de cánceres en áreas rurales son menores que en las zonas urbanas, sin embargo algunos tipos de cánceres se encuentran con mayor incidencia en el mundo agrícola 12 , como son los linfomas no-Hodgkin, los de próstata y los sarcomas, asociados con la fabricación y el empleo de agroquímicos 13

En particular, los clorados persistentes han sido estudiados en relación a diferentes cánceres hormono-dependientes, habiéndose mostrado recientemente una relación con el cáncer de mama 9. Igualmente, un reciente estudio prospectivo, mostró la relación de la utilización de organoclorados con mayor incidencia del cáncer de próstata 14 , independiente de otros factores asociados.

Relacionado con cánceres hormono-dependientes, varios de ellos han sido asociados con el uso de ciertos agro-químicos con acciones de disrupción endocrina: tiroides, ovario, mama, testículo y próstata. Estas asociaciones - vista, sobretodo, en modelos animales y estudios in vitro - requieren de una profunda vigilancia a nivel de poblaciones humanas para poder dilucidar hasta dónde estos fenómenos pudieran ocurrir en ellas 1 .

Finalmente, otros de los hechos que han llamado la atención ha sido el mayor nacimiento de mujeres en relación a los nacimientos de varones, fenómeno también relacionado con factores ambientales 15 .

En los últimos cincuenta años, las actividades agro-productivas en América Latina se han incrementado debido a nuevas tecnologías, nuevos agro-químicos y nuevos cultivos, como los llamados transgénicos. Este proceso ha resultado en un significativo aumento en la productividad, así como, en profundos cambios en los modos de producción, particularmente para el hombre, con las modificaciones que esto significa sobre la salud, tanto favorables como desfavorables.

Comparativamente, pocos trabajos de investigación han sido realizados en América Latina sobre la relación de factores ambientales rurales y salud reproductiva, si bien algunos han mostrado la presencia de esta problemática en el subcontinente 16,17,18.

En Argentina, las particulares formas de la producción agropecuaria generalizada en la Pampa Húmeda (Figura 1) han producido una severa exposición - durante varias décadas - a organoclorados, que han permanecido en el ambiente hasta la actualidad, como ha quedado demostrado en suelos 18, así como en productos de consumo diarios 19. Sin dejar de lado la utilización de otros agroquímicos (fosforados, etc.), que han sido utilizados en las décadas del 70 y del 80; incluso actualmente existe un amplio uso de endosulfán y del 2-4D.

En los últimos tiempos, este fenómeno ha cambiado y se ha caracterizado por un $95 \%$ de cultivo de soja transgénica, dando lugar a un fenómeno ampliamente difundido de modificación de las condiciones del ecosistema que, devenido en agroecosistema de gran magnitud, ofrece cambios sustanciales en su estructura y su función, con alto grado de modificación de los parámetros ambientales básicos, como modificaciones en su estructura y función: la pérdida de biodiversidad y erosión genética, simplificación del ecosistema, simplificación de cadenas y redes tróficas, introducción de moléculas sintéticas (biocidas y fertilizantes, contaminantes y persistentes, que se incorporan a los ciclos biogeoquímicos), pérdida en calidad de suelos (degradación físico-química y pérdida de estructura) 20. 
El objetivo general del presente trabajo intenta describir situaciones de salud reproductiva en comunidades rurales, en la zona sur de la Pampa Húmeda Argentina.

\section{Material y métodos}

\section{Descripción del área geográfica}

El enfoque metodológico para la selección del área geográfica aplicado es como se detalla a continuación.

Selección de las áreas consideradas representativas del modelo agro-productivo predominante en la Pampa Húmeda Argentina (Figura 1). Las localidades fueron seleccionadas bajo el criterio de que constituyeran poblaciones consideradas rurales, con cinco mil habitantes o menos, ubicadas en la zona central del área de mayor incidencia del modelo productivo actual de soja y con antecedentes de haber estado dedicadas a la producción agropecuaria al menos desde la década de 1950.

La información estadística poblacional del Instituto Nacional de Estadísticas y Censos e Instituto Provincial de Estadísticas y Censos (IPECINDEC) fue de donde se obtuvieron datos generales censales (http://www.indec.mecon.ar).

\section{Comunidades participantes}

Dentro de la zona descrita se seleccionaron cinco comunidades rurales según sus correspondientes poblaciones totales (Censo 2001; http:/ / www. indec.mecon.ar): Alcorta $(\mathrm{n}=5.000)$, Bigand $(\mathrm{n}=4.998)$, Carreras $(\mathrm{n}=2.137)$, Máximo Paz $(\mathrm{n}=$ 3.552) y Santa Teresa $(\mathrm{n}=3.278)$.

Las mencionadas localidades han sido escogidas sobre la base de tres criterios de inclusión: áreas altamente explotadas en los últimos 50 años por diferentes actividades agro-productivas y, por lo tanto, expuestas a diferentes agro-químicos; poblaciones con menos de 5.000 habitantes; y, facilitación de acceso comunitario, debido a la inserción colaborativa de la Federación Agraria Argentina.

\section{Variables a ser estudiadas}

El diseño es de tipo descriptivo cuya finalidad es describir la incidencia de diversas variables de salud reproductiva. El presente modelo no permite estructurar relaciones de causalidad.

Se evaluaron tres diferentes variables de salud rural: índice de proporciones de nacimientos masculinos y femeninos; incidencia de malformaciones uro-genitales masculinas (testículos
Figura 1

Mapa de la zona geográfica de estudio.

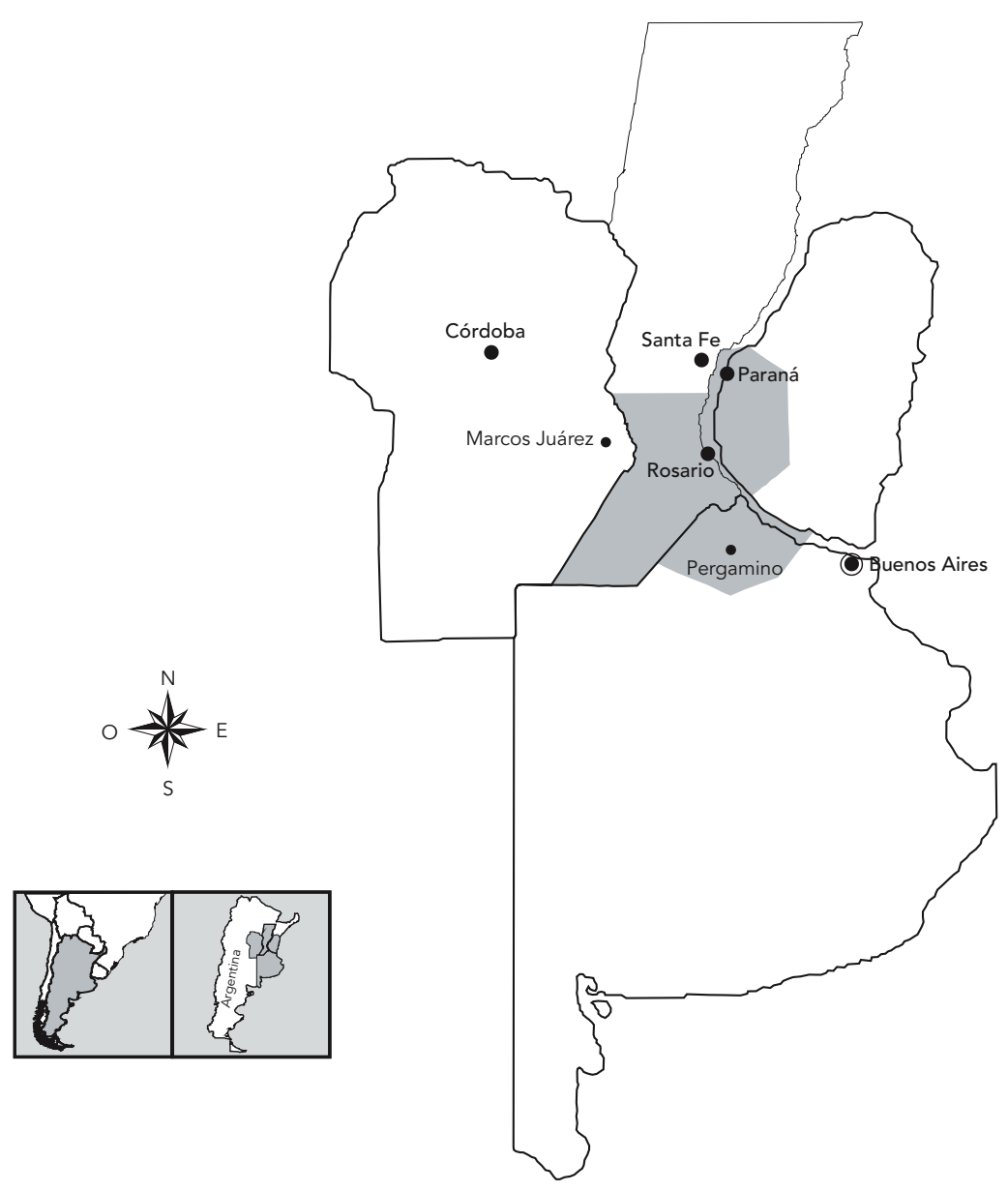

no descendidos e hispospadias); e incidencia de cánceres hormono-dependientes (mama, testículo, próstata y ovario).

\section{Fuentes de información bio-médica}

En Argentina existen pocos datos de registros de salud confiables, sólo en algunas fuentes locales pueden encontrarse registros parciales de los mismos.

En el caso de las cinco comunidades, se analizaron los datos de los Servicios de Asistencia Médica Comunitarios (SAMCO), creados durante la década de los 70, que cubren asistencialmente a aproximadamente los dos tercios del total de la población de las cinco comunidades seleccionadas. El tercio restante tiene cobertura privada, y 
se atiende en centros de grandes ciudades de la región, sin posibilidad de acceso a información retrospectiva.

El cálculo de los dos tercios del total de población fue extraído de los registros existentes, utilizando número de consultas de primera vez, así como la distribución geográfica de los habitantes que consultaron. Las características socioeconómicas de la población de consulta se encontraban registradas, y con ello se pudo evaluar la representatividad de los mismos en el contexto de la población general de cada comunidad, lo cual fue documentado en otra publicación de este mismo proyecto 21 .

La proporción de nacimientos por sexos (masculino/femenino) por comunidades fue realizada sobre datos censales del año 2001, considerando los nacimientos ocurridos en el período 1991-2001. Este Censo muestra la distribución por edades de los primeros 10 años de vida, con lo cual puede obtenerse la sumatoria de los nacimientos ocurridos en cada comunidad, considerando que la migración de estas poblaciones es casi nula, como lo demuestran los registros comunales.

Las malformaciones uro-genitales masculinas fueron realizadas sobre datos obtenidos de los SAMCO de Carreras (1995-2005) y Máximo Paz (1999-2004), debido a que las demás comunidades no contaban con la mencionada información. Los datos registrados son aquellos que fueron detectados en la primera revisión médica obligatoria de los niños, a la edad de 1 año.

En el caso de la recolección de datos sobre cánceres se obtuvo la información de los casos registrados durante el año 2003, ajustados por datos censales de los períodos 1991 y 2001 proyectados, para establecer el número de casos por grupo etario, tomando en consideración sexo y edad en intervalos de cinco años.

\section{Análisis estadístico}

La proporción de nacimientos fue realizada utilizando un cálculo de total de nacimientos para los períodos mencionados, expresado como el número de nacimientos masculinos por cada 100 femeninos.

Con el fin de comparar los mismos con cifras generales de la región se utilizaron los nacimientos registrados, durante el mismo período la media de la provincia de Santa Fe, a la cual pertenecen los pueblos estudiados.

Para fines de los análisis comparativos, el test de chi-cuadrado fue utilizado para las variables categorizadas. El análisis fue realizado utilizando un paquete de software llamado Statview (SAS Inst., Cary, Estados Unidos). Todos los tests fue- ron bilaterales y los valores de $\mathrm{p}<0,05$ fueron considerados significativos.

Las malformaciones uro-genitales masculinas fueron divididas en testículos no descendidos y hipospadias, y calculadas sobre el total de nacimientos masculinos, obteniéndose el valor de incidencia para los períodos registrados. Se debe aclarar que en el caso de las hipospadias se incluyeron sólo aquellas llamadas verdaderas, excluyéndose tanto las epispadias como las balánicas.

Los datos fueron comparados en el caso de testículos no descendidos, con la media de incidencia de la Comunidad Europea. Mientras que, en el caso de las hipospadias, se utilizaron los registros de la misma Comunidad Europea 22, y de la registrada por el Estudio Colaborativo Latinoamericano de Malformaciones Congénitas (ECLAMC), registros de la Ciudad de Rosario, 1982-1992 23

En el caso de los cánceres, con los datos obtenidos del mencionado período 2003, se realizaron análisis por comunidades y sobre el total de la población bajo estudio.

Tasa de incidencia cruda y de incidencia estandarizada a la distribución de la edad de la población estándar mundial fueron calculadas como se describe 24

Datos de incidencia para las ciudades de Concordia y Bahía Blanca, cubriendo el año 2002, y la información registrada en GLOBOCAN 200225 para datos estimados de Argentina para el mismo año se utilizaron para la comparación.

\section{Resultados}

En el total de las comunidades se observa la misma proporción de nacimientos masculinos y femeninos que en el total de la Provincia de Santa Fe $(p=0,91)$.

Como puede verse en la Tabla 1, la proporción de nacimientos presenta en sólo dos comunidades (Carreras y Santa Teresa) una leve tendencia, no significativa, a mayores nacimientos masculinos.

Dentro de las malformaciones uro-genitales masculinas, en el caso de Carreras, se obtuvieron registros de 11 años (1995-2005), con un total de 189 varones nacidos en ese período. Se observó una incidencia media sobre el periodo considerado del $16.4 \%$ para testículos no descendidos; mientras que en hipospadia, con una incidencia del $2 \%$.

Para Máximo Paz, se analizaron registros existentes de los últimos 6 años (1999-2004), totalizando 162 nacimientos masculinos. En el caso de testículos no descendidos, la incidencia 
Relación de nacimientos femeninos/masculinos en el período 1991-2001.

\begin{tabular}{|c|c|c|c|c|c|c|}
\hline \multirow[t]{2}{*}{ Comuna } & \multicolumn{2}{|c|}{ Varones } & \multicolumn{2}{|c|}{ Mujeres } & \multirow[t]{2}{*}{$\chi^{2}$} & \multirow[t]{2}{*}{$p$} \\
\hline & $\mathbf{n}$ & $\%$ & $\mathbf{n}$ & $\%$ & & \\
\hline Santa Fe (totales de la provincia) & 261.940 & 50,74 & 254.345 & 49,26 & - & - \\
\hline Bigand & 352 & 51,24 & 335 & 48,76 & 2,035 & 0,154 \\
\hline Máximo Paz & 218 & 49,21 & 225 & 50,79 & 0,085 & 0,770 \\
\hline Carreras & 169 & 53,99 & 144 & 46,01 & 3,759 & 0,053 \\
\hline Santa Teresa & 253 & 52,38 & 230 & 47,62 & 3,027 & 0,059 \\
\hline Alcorta & 530 & 49,07 & 550 & 50,93 & 0,134 & 0,714 \\
\hline
\end{tabular}

media es del 6,2\%, mientras que para hipospadia es de $1,4 \%$.

En el caso de los diferentes cánceres hormono-dependientes, como lo indica la Tabla 2, se destacan los cánceres de testículo y ovario, mostrando un incidencia tres veces mayor en el primer caso, y de casi dos veces en el segundo, comparados con las incidencias obtenidas en los registros de Concordia, Bahía Blanca y las estimaciones para Argentina obtenidas del GLOBOCAN 2002 25. Mientras que los cánceres de próstata y mama femenino se encuentran por debajo de los registrados en las medias nacionales. Cuando se analizan las situaciones individuales para cada comuna se pueden observar diferentes a las generales mencionadas, no obstante, el escaso número de casos encontrados por comunas no permiten establecer una evaluación confiable.

En relación con los dos cánceres hormonodependientes, que mostraron menor incidencia (próstata y mama) que las medias comparativas, existen dos excepciones: en el caso de Carreras se observa una mayor presencia de cáncer de mama femenino. Así como, en Máximo Paz, aparece destacándose el de próstata, con un $50 \%$ más de los registrados en las incidencias usadas para la comparación.

\section{Discusión}

El objetivo de este trabajo era el de describir los datos existentes en las variables reproductivas seleccionadas, comparándolo con los registros generales, con el fin de evaluar las inquietudes y percepciones presentadas por las poblaciones. Este trabajo ni intenta ni permite establecer una relación causal con factores explicativos, lo cual será motivo de otro estudio que permita establecer la posible relación existente entre ellos.

Inicialmente, debe aclararse que los presentes resultados deben ser interpretados con cautela, debido al número de datos limitado, así como a la forma de registro que se han utilizado como base documental de este proyecto.

En relación con la proporción de nacimientos masculino/femenino, estos datos no presentan ningún sesgo dada la exhaustividad obtenida a partir del Censo 2001.

Con respecto a las malformaciones, las fuentes de información fueron limitadas a dos de las cinco comunidades estudiadas, las únicas que disponen de registro de examen médico obligatorio de los nacidos vivos antes del año. Este examen permite de excluir las remisiones espon-

Tabla 2

Totales de incidencia de cánceres para el año de 2003.

\begin{tabular}{lcccc}
\hline Tipo & $\begin{array}{c}\text { Concordia } \\
\text { (año 2002) }\end{array}$ & $\begin{array}{c}\text { Bahía Blanca } \\
\text { (año 2002) }\end{array}$ & $\begin{array}{c}\text { Argentina } \\
\text { (GLOBOCAN 2002 25) }\end{array}$ & $\begin{array}{c}\text { Encontrada } \\
\text { (total de comunidades, año 2003) }\end{array}$ \\
\hline Próstata & 31,3 & 31,9 & 36,8 & 25,1 \\
Mama (femenino) & 55,1 & 86,1 & 73,9 & 47,9 \\
Testículo & 2,2 & 3,6 & 4,2 & 15,2 \\
Ovario & 8,3 & 9,1 & 8,0 & 14,2 \\
\hline
\end{tabular}


táneas de testículos no descendidos, así como los fallecidos al nacer y durante el año siguiente.

Finalmente, los datos sobre cánceres se han podido establecer sobre la base de los registros, que solamente cubren dos tercios de la población, estimándose no sólo la posibilidad de subregistros con relación a la totalidad de las patologías, sino a la especificidad por tipo de cánceres, que podrían llevar a la sobre o sub-estimación en muchos de ellos.

Respecto de las tres variables seleccionadas, en la primera de ellas - la relación de nacimientos masculinos/femeninos - no se observan valores diferentes de los habituales para la región, particularmente en comparación con la provincia a la cual pertenecen las comunidades bajo estudio.

Mucho se ha discutido sobre la influencia de los ambientes rurales sobre el mayor índice de nacimientos femeninos en humanos, en ello sólo se encontró una relación directa con la presencia de dioxinas en poblaciones ampliamente expuestas 26; factor ambiental de mediano impacto en poblaciones rurales, debido a la poca industrialización de estas regiones 27 .

Cuando se analizan los resultados de las malformaciones uro-genitales masculinas, las cifras son más elocuentes al respecto, ya que las incidencias se ven amplificadas entre 10 para testículos no descendidos y alrededor de 20 veces para hipospadias, al menos en las dos comunidades donde se obtuvieron datos cuando son comparadas con datos europeos o de registros regionales. En ambas malformaciones, testículos no descendidos e hipospadias, existen datos en el mundo de un incremento significativo de la incidencia de estas patologías en poblaciones generales en los últimos 50 años 28 , aunque cuando se consideran las poblaciones rurales en particular, los datos son controvertidos, dependiendo de las regiones del mundo que se consideren. Estas cifras exigen reanalizar los casos y, preferencialmente, llevar adelante estudios de prevalencia en población escolar, que permitan confirmar este fenómeno.

En lo relacionado con la incidencia de cánceres, existe una importante discusión en la literatura sobre el aumento de incidencias de aquellos denominados hormono-dependientes 29 .
En el caso particular de los presentes datos, se observa que en el análisis general de las comunidades que se incluyeron, y dentro de los cánceres hormono-dependientes, se destacan el de testículo y ovario por una mayor incidencia; así como los de próstata y mama femenino, con una incidencia menor, cuando fueron comparados con los otros registros.

Al considerar las diferencias existentes, en estos datos y los generales de la región, no pueden dejarse de lado los factores alimenticios de la zona bajo análisis (dietas ricas en proteínas y sal), que podrían influenciar la mayor incidencia mencionada, sin dejar de lado lo relacionado con el tabaquismo; o quizás la sumatoria de todos estos factores que debería ser considerada. Igualmente factores genéticos y de predisposición endogámica, por las características de estas poblaciones de pocos habitantes.

Quizás exposiciones regionales a pasados agro-químicos del tipo de los clorados han influenciado estas circunstancias, así como la presencia de otros factores (arsénico, nitritos) que podrían existir en mayor cantidad en estas zonas.

En otra publicación del presente proyecto 30 se presenta la ubicación geográfica de los diferentes casos sobre un sistema de geo-referenciación, que permite ubicar las diferentes fuentes fijas de posible riesgo ambiental, lo cual ofrece datos complementarios para la identificación de este impacto, observándose que más del $90 \%$ de los casos se encuentran ubicados a menos de 300 metros de las mencionadas fuentes sospechosas.

Finalmente, y a modo de síntesis, este trabajo destaca dos riesgos potenciales en estas poblaciones rurales, relacionados con malformaciones y cánceres hormono-dependientes.

El hecho de identificar el riesgo no significa que pueda inferirse la magnitud del mismo, sino simplemente y cautelosamente - por las razones antes expuestas - hace necesario entrar en una segunda fase que es la del "diagnóstico del riesgo", que requiere de una mayor precisión en términos de historias de salud y de diagnósticos eco-sistémicos relacionados (marcadores ambientales y poblacionales), permitiendo así poder confirmar los resultados aquí encontrados. 


\section{Resumen}

La relación entre factores ambientales y salud es un hecho reconocido. La influencia de ambientes rurales sobre la salud reproductiva ha sido fehacientemente probada en diferentes regiones del mundo, tanto en la fauna como en humanos. En América Latina pocas investigaciones han sido realizadas en este campo. El presente proyecto se establece sobre la base de la describir las relaciones entre salud reproductiva y factores ambientales en poblaciones rurales, caracterizada por aspectos ambientales particulares. Tres variables han sido evaluadas: relación de nacimientos masculinos/femeninos; incidencia de malformaciones urogenitales masculinas (hipospadias y criptorquidias); e incidencia de cánceres hormono-dependientes. Se seleccionaron cinco comunidades rurales de la Pampa Húmeda de Argentina, comparándose los datos obtenidos con medias nacionales. Los datos bio-médicos y las fuentes ambientales de riesgo fueron relacionados entre sí a través de un sistema de geo-referenciación. La relación de nacimientos no mostró significación. Las malformaciones presentaron una muy significativa incidencia. Los cánceres hormono-dependientes presentaron incidencia mayores a las medias nacionales, particularmente en algunas de las comunidades estudiadas. Se concluye que existe una relación entre condiciones de salud reproductiva y factores ambientales en esta región.

Salud Reproductiva; Medio Ambiente y Salud Pública; Población Rural

\section{Colaboradores}

A. Oliva estuvo a cargo de la recolección y análisis de los datos bio-médicos. R. Biasatti se ocupó de la selección de las poblaciones, así como de las evaluaciones ecosistémicas y el desarrollo del GIS. A. Gelin y S. Olego estuvieron a cargo de la logística de relaciones comunitarias. S. Cloquell cubrió los aspectos sociológicos. C. González realizó los análisis estadísticos.

\section{Agradecimientos}

El presente proyecto ha sido financiado por el Programa VIGI+A del Ministerio de Salud y Ambiente de la Nación, República Argentina (proyecto no. 1055). Al Dr. Luc Multigner (INSERM U 625, Universitè du Rennes I, Francia), por su valiosa colaboración para la preparación del presente trabajo.

\section{Referencias}

1. Cocco P. On the rumors about the silent spring: review of the scientific evidence linking occupational and environmental pesticide exposure to endocrine disruption health effects. Cad Saúde Pública 2002; 18:379-402

2. U.S. Environmental Protection Agency. Special report on environmental endocrine disruption: an effects assessment and analysis. Washington DC: U.S. Environmental Protection Agency; 1997.

3. Arcury TA, Quandt SA, Dearry A. Farmworker pesticide exposure and community-based participatory research: rationale and practical applications. Environ Health Perspect 2001; 109 Suppl 3:429-34.

4. Royal Commission on Environmental Pollution. Crop spraying and the health of residents and bystanders. London: Royal Commission on Environmental Pollution; 2005.

5. Carpenter DO, Arcaro K, Spink DC. Understanding the human health effects of chemical mixtures. Environ Health Perspect 2002; 110 Suppl 1:25-42.
6. Stang A, Ahrens W, Bromen K, Baumgardt-Elms C, Jahn I, Stegmaier C, et al. Undescended testis and the risk of testicular cancer: importance of source and classification of exposure information. Int J Epidemiol 2001; 30:1050-6.

7. Oliva A, Spira A, Multigner L. Contribution of environmental factors to the risk of male infertility. Hum Reprod 2001; 16:1768-76.

8. Oliva A, Giami A, Multigner L. Environmental agents and erectile dysfunction: a study in a consulting population. J Androl 2002; 23:546-50.

9. Snedeker SM. Pesticides and breast cancer risk: a review of DDT, DDE, and dieldrin. Environ Health Perspect 2001; 109 Suppl 1:35-47.

10. Pauwels A, Schepens PJ, D'Hooghe T, Delbeke L, Dhont M, Brouwer A, et al. The risk of endometriosis and exposure to dioxins and polychlorinated biphenyls: a case-control study of infertile women. Hum Reprod 2001; 16:2050-5. 
11. Krstevska-Konstantinova M, Charlier C, Craen M, Du Caju M, Heinrichs C, Beaufort C, et al. Sexual precocity after immigration from developing countries to Belgium: evidence of previous exposure to organochlorine pesticides. Hum Reprod 2001; 16:1020-6.

12. Rautiainen RH, Reynolds SJ. Mortality and morbidity in agriculture in the United States. J Agric Saf Health 2002; 8:259-76.

13. Blair A, Zahm SH. Agricultural exposures and cancer. Environ Health Perspect 1995; 103 Suppl 8:205-8.

14. Alavanja MC, Samanic C, Dosemeci M, Lubin J, Tarone R, Lynch CF, et al. Use of agricultural pesticides and prostate cancer risk in the Agricultural Health Study cohort. Am J Epidemiol 2003; 157:800-14.

15. Davis DL, Gottlieb MB, Stampnitzky JR. Reduced ratio of male to female births in several industrial countries: a sentinel health indicator? JAMA 1998; 279:1018-23.

16. Restrepo M, Muñoz N, Day N, Parra JE, Hernan$\operatorname{dez} \mathrm{C}$, Blettner M, et al. Birth defects among children born to a population occupationally exposed to pesticides in Colombia. Scand J Work Environ Health 1990; 16:239-46.

17. Tortolero I, Bellabarba-Arata G, Lozano R, Bellabarba C, Cruz I, Osuna J. Semen analysis in men from Merida, Venezuela, over a 15-year period. Arch Androl 1999; 42:29-34.

18. Garcia-Fernandez J, Casabella C, Marzi A, Astolfi E, Roses O, Donnewald H, et al. Organochlorinated pesticides in the Argentine Antarctic sector and Atlantic coastline waters. Geogr Med 1979; 9:28-37.

19. Maitre M, De La Sierra P, Lenardon A, Enrique S, Marino F. Pesticide residue levels in Argentinean pasteurized milk. Sci Total Environ 1994; 155: 105-8.

20. Viglizzo E, Roberto Z, Brockington N. Análisis del impacto de componentes ambientales y empresariales sobre el comportamiento de agroecosistemas de la Pampa Semiárida Argentina. Revista Argentina de Producción Animal 1992; 12:319-29.
21. Cloquell S, González C, Biasatti R, Oliva A. Identificación de relaciones entre salud rural y exposiciones a factores ambientales en la Pampa Húmeda Argentina. Parte 3. Aspectos Sociales. In: VII Congreso Latinoamericano de Sociología Rural. Quito: Facultad Latinoamericana de Ciencias Sociales; 2006. p. 844-5.

22. World Health Organization. World atlas of birth defects. 2nd Ed. Geneva: World Health Organization; 2003.

23. Castilla EE, Lopez-Camelo JS, Paz JC. Atlas geográfico de las malformaciones congénitas en Sudamérica. Argentina, Región Centro. 1ạ Ed. Río de Janeiro: Editora Fiocruz; 1995.

24. Bray F. Age-standardization: cancer incidence in five continents. v. VIII. Lyon: IARC Press; 2002. (IARC Scientific Publications, 155).

25. Ferlay J, Bray F, Pisani P, Parkin DM. GLOBOCAN 2002: cancer incidence, mortality and prevalence worldwide. Lyon: IARC Press; 2004.

26. Mocarelli P, Gerthoux PM, Ferrari E, Patterson Jr. DG, Kieszak SM, Brambilla P, et al. Paternal concentrations of dioxin and sex ratio of offspring. Lancet 2000; 355:1858-63.

27. Ryan JJ, Amirova Z, Gaetan Carrier G. Sex ratios of children of Russian pesticide producers exposed to dioxin. Environ Health Perspect 2002; 110: A699-701.

28. Dolk H, Vrijheid M, Armstrong B, Abramsky L, Bianchi F, Garne E, et al. Risk of congenital anomalies near hazardous-waste landfill sites in Europe: the EUROHAZCON study. Lancet 1998; 352:423-7.

29. Birnbaum LS, Fenton SE. Cancer and developmental exposure to endocrine disruptors. Environ Health Perspect 2003; 111:389-94.

30. Biasatti NR, Oliva A, Cloquell S, González C, Olego S, Gelin A. Agrosistema de la Pampa Húmeda (Argentina): relaciones entre factores ambientales de riesgo y la salud de la población rural. Parte 2. Aspectos ecosistémicos. In: 8as Jornadas Rosarinas de Antropología Sociocultural. Rosario: Escuela de Antropología, Facultad de Humanidades y Artes, Universidad Nacional de Rosario; 2006. p. 1-11.

Recibido el 28/Mar/2006

Versión final presentada el 18/Ene/2007 Aprobado el 24/Sep/2007 